\title{
Avian reticuloendotheliosis in chickens - an update on disease occurrence and clinical course
}

\author{
Grzegorz Woźniakowski ${ }^{1}$, Maciej Frant ${ }^{1}$, Andrzej Mamczur ${ }^{2}$ \\ ${ }^{1}$ Department of Swine Diseases, National Veterinary Research Institute, 24-100 Pulawy, Poland \\ ${ }^{2}$ Avi-Vet Service, Mamczur J., Szeptycki S.J., 62-080 Tarnowo Podgórne, Poland \\ grzegorz.wozniakowski@piwet.pulawy.pl
}

Received: April 17, $2018 \quad$ Accepted: August 22, 2018

\begin{abstract}
Avian reticuloendotheliosis (RE) represents an important immunosuppressive disease of poultry. The occurrence of RE in both chickens and turkeys has an immunosuppressive effect and may lead to vaccination failures. Avian reticuloendotheliosis virus (REV) is widely distributed in different kinds of birds, causing subclinical infections. Another important issue adhering to this disease is contamination of vaccines against fowl pox (FP) and Marek's disease (MD) with REV. The capability of REV to integrate into the genome of other larger DNA viruses complicates its diagnosis and prevention. There are no efficient vaccines against RE nor treatment, which also complicates how to limit its impact on poultry farming. This paper reviews the current state of knowledge of this important immunosuppressive agent of poultry emphasising the importance of this problem in terms of diagnosis of RE.
\end{abstract}

Keywords: chickens, avian reticuloendotheliosis, avian reticuloendotheliosis virus, disease course, diagnosis.

\section{Introduction}

Avian reticuloendotheliosis (RE) is a malignant disease of poultry most frequently affecting chickens and turkeys but also waterfowl such as ducks and geese as well as other bird species $(10,16,26)$. The clinical course of RE may be similar to other neoplastic diseases including Marek's disease (MD), lymphoid leukosis (LL), or avian leukosis caused by avian leukosis virus of subgroup $\mathrm{J}$ (ALV-J) $(2,3,11)$. The mortality may reach approximately $16 \%$ in chickens, dependening on the age of the birds (25). The most common outcome of the disease in chickens is growth retardation and runting syndrome (26). From the histopathological point of view, the disease leads to acute or chronic neoplasia of lymphoid tissues. The occurrence of the disease has major economic importance, also taking into account the virus transfer to young chickens during vaccination with a contaminated preparation (35). The emergence of a sole $\mathrm{RE}$ is a rather rare phenomenon. A previous study on the incidence of RE in chicken flocks conducted in Poland raised concerns regarding the contamination of vaccines against fowl pox (FP) or MD with reticuloendotheliosis virus (REV) strains (20). The only preventive measure is testing vaccine batches against $\mathrm{MD}$ and FP for potential retrovirus contamination. Moreover, the RE in the vaccine may lead to failure in vaccination against other infectious diseases including MD or FP $(1,8,17)$. It has been also shown that co-incidence of RE with other secondary infectious agents may lead to increased rate of tumour formation in chickens (22). So far, no effective vaccines have been developed against $R E$, thus the only protection remains flock renewal with elimination of affected birds or application of experimental antiviral treatment (26). One of the main pillars of RE elimination is efficient laboratory diagnosis $(1,35)$.

Transmissions and vectors. Infection with REV may be transmitted by a horizontal route by direct contact between birds, indirectly by some insect vectors like mosquitos (Culex pipiens L) or flies (Musca domestica $L$ ), and also by a vertical route by eggs $(7,23,32)$. The virus has also been experimentally 
recovered from the ticks Triatoma infestans and Ornithodoros moubata (31), but attempts to propagate $\mathrm{REV}$ in cell cultures derived from the mosquito Aedes albopictus were unsuccessful (7). The contribution of contaminated fomites or environments, including poultry houses or pens, should also be taken into account in any epidemiological investigation. Inefficient disinfection may lead to the persistence of REV particles in litter or residues of avian body fluids at the farm. The risk of REV transmission is extant in spite of the virus being rather labile since its virions are rapidly inactivated outside the host (24).

Virus features. Avian REV is a member of the Gammaretrovirinae subfamily within the Retroviridae family. The list of other members of this family includes murine leukaemia virus (MLV), feline leukaemia virus (FeLV), gibbon ape leukaemia virus (GALV), and xenotropic murine leukaemia virusrelated virus (XMRV) (24). REV differs from other retroviruses causing lymphoid leukosis (LL) or avian leukosis subgroup $\mathrm{J}$ (AL-J) because of the genomic structure and mechanism of cell ingress (26). In terms of zoonotic potential of REV, it has been shown that the virus shares some evolutionary lineage with mammalian retroviruses, but the potential to infect humans is negligible (12). In spite of the possible seroconversion in humans and low titres of antibodies present in human sera the infection is non-productive. The virus is represented by a sole serotype which may be differentiated into three antigenic subtypes. The capsid of REV is approximately $100 \mathrm{~nm}$ in diameter. Its density determined on a sucrose density gradient is between 1.16 and $1.18 \mathrm{~g} / \mathrm{mL}$. The genome comprises single stranded RNA (ssRNA), and on the basis of genomic structure two types of REV are distinguished. The genome of non-defective REV-A is $9 \mathrm{kbp}$ long, while the defective REV-T strain is $3.3 \mathrm{kbp}$ shorter due to the deletion between the envelope gene (env), groupspecific antigen gene (gag), and RNA dependent DNA polymerase (pol) junctions (Fig. 1). The REV-T genome contains the $\mathrm{v}$-rel oncogene which is missing within the REV-A genome. The consequence of deletions within the defective REV-T genome is the inability to replicate without 'helper' REV-A virus (18). Oncogenic $\mathrm{v}-$ rel is expressed in transformed lymphoid cells as pp59v-rel oncoprotein. This gene belongs to the rel/dorsal protein family related to nuclear factor kappa B involved in binding of DNA transcription factors. REV-T is responsible for an acute form of neoplasia in infected cells. The regions of long terminal repeats (LTRs) are 569 bp long and harbour promoter sites for cell machinery in a number of cell lines (22). These LTR sequences act as a promoter and enhancer for viral RNA translation. So far, at least two complete field strain sequences of the REV genome originating from the United States and China have been submitted to the GenBank database (GenBank accessions: NC006934 and DQ387450).

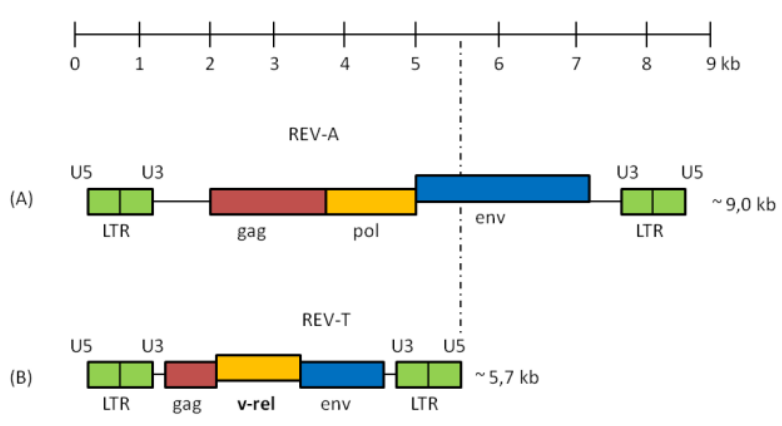

Fig. 1. The genomic structure of REV. (A) - REV-A strain, (B) - "defective" REV-T strain. LTR - long terminal repeats, U3 and U5 - unique regions, gag - gene encoding viral structural protein, pol - RNA-dependent DNA polymerase and DNA integrase, v-rel oncogene present only in the defective REV-T strain, $\beta$ - one thousand base pairs

Virus replication in vitro. The propagation of REV is possible in chicken embryo fibroblasts (CEFs) or duck embryo fibroblasts (DEFs), and in a couple of other avian-origin cell lines (35). The cytopathic effect (CPE) formed by the virus is rather discrete with formation of multi-form syncytia. The virus is also known to propagate in rat kidney cells, mink lung cells, bovine cells, D17 dog sarcoma cells, and Cf2th dog thymus cells (27). The efficient plaque assay has been described by Cho (4) in a chemically transformed QT35 line derived from Japanese quail fibroblasts. The previous attempts at REV propagation in human-origin cells were inefficient probably due to the virus' inability to bind to the surface receptor (12).

Virus integration. REV possesses an ability to integrate proviral DNA into the genome of larger DNA viruses, including Marek's disease virus (MDV) or fowl pox virus $(8,15,20,17,22,29,30,33)$. We have previously shown that these recombination events in LTR regions may lead to emergence of new MDV field isolates with different pathogenicity (35). The whole REV genome insertion has also been found in Gallid herpesvirus 3 (GaHV-3), also known as herpesvirus of turkey (HVT) $(5,6,18)$. In a previous study, Wei et al. (32) showed potential REV contamination of CVI988/Rispens and ND-LaSota+IB-H120 vaccine stocks and congenital transmission of REV among chickens.

Incidence of RE. RE incidence has been described in flocks of chickens, ducks, and turkeys in different countries. The seroprevalence was more frequent in older bird flocks, including layer or breeder chickens. The current status in terms of RE occurrence in the US is declared as common (21). Similar to those findings, Cheng et al. (3) showed $42.6 \%$ incidence of REV among chickens in China. A parallel survey has been conducted by Buscaglia (2), showing that $95 \%$ of flocks in Argentina were seropositive (2). Also in Egypt, a previous examination of chicken flocks revealed its occurrence in broilers (9). Our previous study conducted in Poland indicated the occurrence of 
REV RNA in 24/25 (96\%) of examined chicken flocks infected with MDV (35).

Clinical course. The only specific clinical observation may be abnormal feathering of affected birds called "nakanuke syndrome" (Figs 2A and 2B). $\mathrm{RE}$ has an immunosuppressive influence on the host immune system by abrogation of $\mathrm{T}$ or B lymphocytes and endotheliocytes function (10). Due to the main influence on the host immune system, the disease causes dysfunction of the spleen and atrophy of the bursa of Fabricius and thymus (26). Other lesions might also be observed, including abnormal proventriculus, enteritis, or spleen and liver necrosis (Fig. 3). Infrequently, ulceration of the proventriculus might be observed in cases of infection with oncogenic REV-T strains. It has been reported that nerve lesions as enlargements could be observed in the case of absence of other tumorous diseases, e.g. MD or LL. The origin of REV-induced tumours is associated with B-cells. This has been concluded on the basis of identification by IgM and bursectomy (19).

(A)

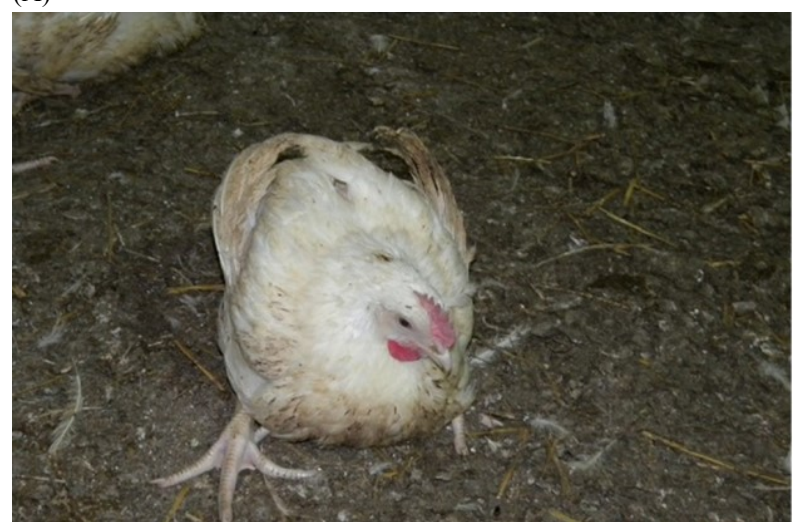

(B)

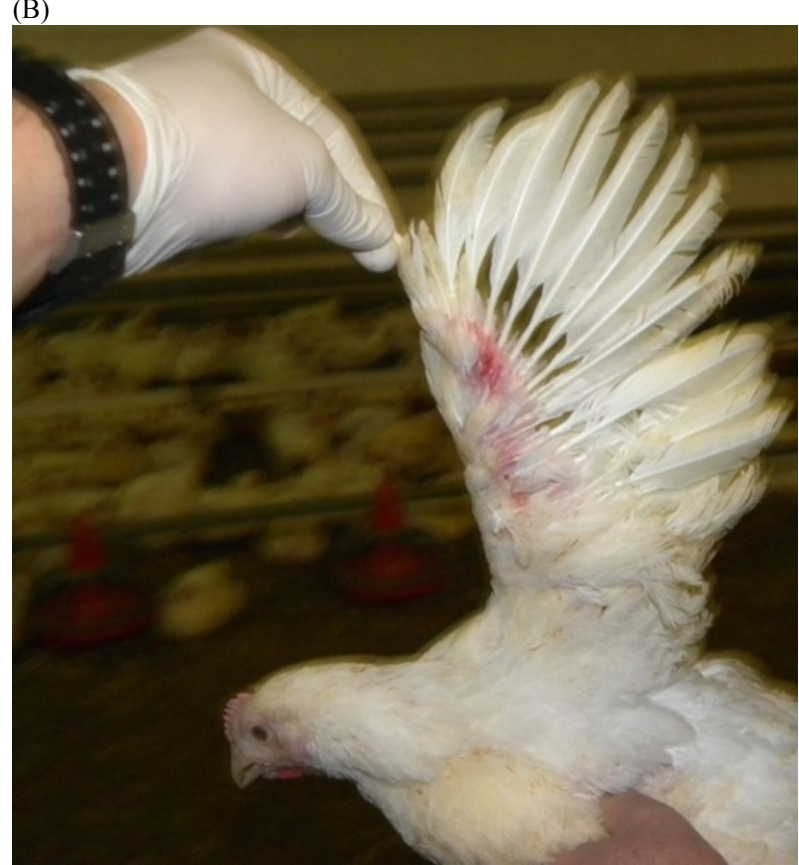

Fig. 2. Nakanuke syndrome of abnormal featheriness in chickens. Panels (A) and (B). The pictures were taken by A. Mamczur

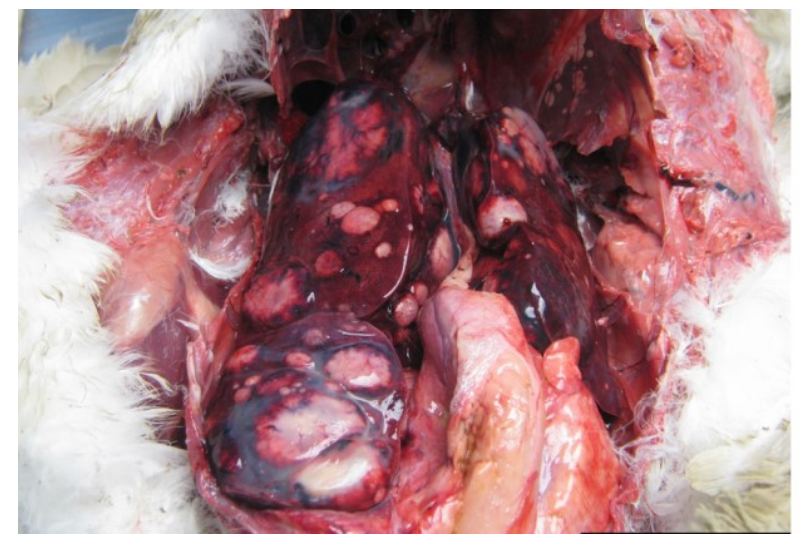

Fig. 3. Lesions and tumours in the liver of a layer chicken caused by REV infection. The picture was taken by A. Mamczur

RE laboratory diagnosis. The current laboratory diagnosis includes REV isolation in CEFs or young nestlings of Japanese quail, geese, ducks, turkeys, pheasants, or guinea fowls. REV might be easily isolated in CEFs, DEFs, or QT35 cell lines. The method could be considered the gold standard and could be used for determination of infectious titres or as a prerequisite for immunofluorescence assays (34). Viraemia of REV is rather low, thus identification of the virus can be difficult. The virus may not produce a reliable cytopathic effect, and an additional two or three passages in cell cultures are recommended. Other laboratory diagnostic methods include the agar gel diffusion precipitation test (AGDT) (13), enzymelinked immunosorbent assay (ELISA) (14), immunofluorescence (34), the complement fixation test (28), RT-PCR, and RT-LAMP assays (35). The most sensitive are immunofluorescence, PCR, or LAMPbased assays. The targets for identification of REV proviral DNA include the pol gene and different LTR fragments. In an alternative approach, virus neutralisation or immunoperoxidase assays offer an robust method of identification of sero-positive animals.

Concluding the present comments on the influence of RE on the economy of poultry production, the most important issue remains the possible contamination of vaccine stocks with REV. Taking into account the integratory properties of this retrovirus, further epidemiological investigation and efficient diagnosis might be difficult or at least inconclusive. Further indepth in vivo studies in different avian species are required to draw firm conclusions regarding the role of REV in tumour onset in a variety of birds.

Conflict of Interests Statement: The authors declare that there is no conflict of interests regarding the publication of this article.

Financial Disclosure Statement: The study was supported by the Polish National Science Centre, grant no. 2011/01/B/NZ1/01561. 
Animal Rights Statement: None required.

\section{References}

1. Awad A.M., El-Hamid H.S.A., Rawash A.A.A., Ibrahim H.H.: Detection of reticuloendotheliosis virus as a contaminant of fowl pox vaccines. Poult Sci 2010, 89, 2389-2395.

2. Buscaglia C.: Mixed infections of Marek's disease and reticuloendotheliosis viruses in layer flocks in Argentina. Avian Dis 2013, 57, 569-571.

3. Cheng Z., Zhang H., Wang G., Liu Q., Liu J., Guo H., Zhou E.: Investigations of avian leukosis virus subgroup $\mathrm{J}$ and reticuloendotheliosis virus infections in broiler breeders in China. Isr J Vet Med 2011, 66, 34-42.

4. Cho B.R.: Cytopathic effects and focus formation by reticuloendotheliosis viruses in a quail fibroblast cell line. Avian Dis 1983, 27, 261-270

5. Cui Z., Zhuang G., Xu X., Sun A., Su S.: Molecular and biological characterization of a Marek's disease virus field strain with reticuloendotheliosis virus LTR insert. Virus Genes 2010, 40, 236-243

6. Davidson I., Borenshtain H.: In vivo events of retroviral long terminal repeat integration into Marek's disease virus in commercial poultry: detection of chimeric molecules as a marker. Avian Dis 2001, 45, 102-121.

7. Davidson I., Braverman Y.: Insect contribution to horizontal transmission of reticuloendotheliosis virus. J Med Entomol 2005, 42, 128-133.

8. Diallo I.S., MacKenzie M.A., Spradbrow P.B., Robinson W.F.: Field isolates of fowlpox virus contaminated with reticuloendotheliosis virus. Avian Pathol 1998, 27, 60-66.

9. El-Sebelgy M.M., Ahmed B.M., Ata N.S., Hussein H.A.: Molecular detection and characterization of reticuloendotheliosis virus in broiler breeder chickens with visceral tumors in Egypt. Int J Vet Sci Med 2014, 2, 21-26

10. Etienne L., Emerman M.: The mongoose, the pheasant, the pox, and the retrovirus. PLoS Biol 2013, 11, e1001641.

11. Fadly A.M., Smith E.J.: Isolation and some characteristics of a subgroup J-like avian leukosis virus associated with myeloid leukosis in meat-type chickens in the United States. Avian Dis 1999, 43, 391-400

12. Gautier R., Jiang A., Rousseau V., Dornburg R., Jaffredo T.: Avian reticuloendotheliosis virus strain $\mathrm{A}$ and spleen necrosis virus do not infect human cells. J Virol 2000, 74, 518-522.

13. Ianoconescu M.: Reticuloendotheliosis antigen for agar gel precipitation test. Avian Pathol 1977, 6, 259-267.

14. Ignjatovic J., Fahey K.J., Bagust T.J.: An enzyme-linked immunosorbent assay for detection of reticuloendotheliosis virus infection in chickens, Avian Pathol 1987, 16, 609-621.

15. Isfort R., Jones D., Kost R., Witter R., Kung H.J.: Retrovirus insertion into herpesvirus in vitro and in vivo. Proc Natl Acad Sci USA 1992, 89, 991-995.

16. Khordadmehr M., Firouzamandi M., Zehtab-Najafi M., Shahbazi R.: Naturally occurring co-infection of avian leukosis virus (subgroups $\mathrm{A}-\mathrm{E}$ ) and reticuloendotheliosis virus in green peafowls (Pavo muticus). Braz J Poult Sci 2017, 19, 609-614.

17. Koo B.S., Lee H.R., Jeon E.O., Jang H.S., Han M.S., Min K.C., Lee S.B., Kim J.J., Mo I.P.: An outbreak of lymphomas in a layer chicken flock previously infected with fowlpox virus containing integrated reticuloendotheliosis virus. Avian Dis 2013, 57, 812-817.

18. Li J., Yang C., Li Q., Li H., Xia Y., Liu D., Yu K., Yang H.: Complete genome sequence of reticuloendotheliosis virus strain MD-2, isolated from a turkey herpesvirus vaccine contaminated. Genome Announc 2013, 1, 5, doi:10.1128/genomeA.00785-13.
19. Linna T.J., Hu C.P., Thompson K.D.: Development of systemic and local tumors induced by avian reticuloendotheliosis virus after thymectomy or bursectomy. J Nat Cancer Inst 1974, 53, 847-854.

20. Lupiani B., Lee L.F., Kreager K.S., Witter R.L., Reddy S.M. Insertion of reticuloendotheliosis virus long terminal repeat into the genome of CVI988 strain of Marek's disease virus results in enhanced growth and protection. Avian Dis 2013, 57, 427-431.

21. Mays J.K., Silva R.F., Lee L.F., Fadly A.M.: Characterization of reticuloendotheliosis virus isolates obtained from broiler breeders, turkeys, and prairie chickens located in various geographical regions in the United States. Avian Pathol 2010, 39, 383-389.

22. Mays J.K., Silva R.F., Kim T., Fadly A.M.: Insertion of reticuloendotheliosis virus long terminal repeat into a bacterial artificial chromosome clone of a very virulent Marek's disease virus alters its pathogenicity. Avian Pathol 2012, 41, 259-265.

23. Motha M.X.J., Egerton J.R.: Vertical transmission of reticuloendotheliosis virus in chickens. Avian Pathol 1987, 16, $141-147$.

24. Niewiadomska A.M., Gifford R.J.: The extraordinary evolutionary history of the reticuloendotheliosis viruses. PLoS Biol 2013, 11, e1001642.

25. Okoye J.O., Ezema W., Agoha J.N.: Naturally occurring clinical reticuloendotheliosis in turkeys and chickens. Avian Pathol 1993, 22, 237-244

26. Payne L.N., Venugopal K.: Neoplastic diseases: Marek's disease, lymphoid leukosis, and reticuloendotheliosis. Diseases of Poultry: World Trade and Public Health Implications. Scientific and Technical Review. Off Int Epiz 2000, 19, 564-544.

27. Rice N.R, Hiebsch R.R., Gonda M.A., Bose H.R., Gilden R.V.: Genome of reticuloendotheliosis virus: Characterization by use of cloned proviral DNA. J Virol 1982, 42, 237-252.

28. Smith E.J., Solomon J.J., Witter R.L.: Complement-fixation test for reticuloendotheliosis viruses: limits of sensitivity in infected avian cells. Avian Dis 1977, 21, 612-622.

29. Sun A., Petherbridge L., Zhao Y., Li Y., Nair V.K., Cui Z.: A BAC clone of MDV strain GX0101 with REV-LTR integration retained its pathogenicity. Chin Sci Bull 2010, 54, 2641-2647.

30. Sun A., Xu X., Petherbridge L., Zhao Y., Nair V.K., Cui Z.: Functional evaluation of the role of reticuloendotheliosis virus long terminal repeat (LTR) integrated into the genome of a field strain of Marek's disease virus. Virology 2010, 397, 270-276.

31. Thompson K.D., Fischer R.G., Luecke D.H.: Quantitative infectivity studies of avian reticuloendotheliosis virus (strain T) in certain hematophagous arthropods. J Med Entomol 1971, 8, 486-490.

32. Wei K., Sun Z., Zhu S., Guo W., Sheng P., Wang Z., Zhao C., Zhao Q., Zhu V.: Probable congenital transmission of reticuloendotheliosis virus caused by vaccination with contaminated vaccines. PLoS One 2012, 7, e43422.

33. Witter R.L., Li D., Jones D., Lee L.F., Kung H.J.: Retroviral insertional mutagenesis of a herpesvirus: a Marek's disease virus mutant attenuated for oncogenicity but not for immunosuppression or in vivo replication. Avian Dis 1997, 41, 407-421.

34. Witter R.L., Purchase H.G., Burgoyne G.H.: Peripheral nerve lesions similar to those of Marek's disease in chickens inoculated with reticuloendotheliosis virus. J Nat Canc Inst 1970, 45, $567-577$.

35. Woźniakowski G., Mamczur A., Samorek-Salamonowicz E.: Common occurrence of Gallid herpesvirus-2 with reticuloendotheliosis virus in chickens caused by possible contamination of vaccine stocks. J Appl Microbiol 2015, 118, 803-808. 